\title{
Estimation of Time-Scaling Factor for Ultrasound Medical Images Using the Hilbert Transform
}

\author{
Jérémie Fromageau, ${ }^{1}$ Hervé Liebgott, ${ }^{2}$ Elisabeth Brusseau, ${ }^{2}$ Didier Vray, ${ }^{2}$ and Philippe Delachartre ${ }^{2}$ \\ ${ }^{1}$ Laboratory of Biorheology and Medical Ultrasonics (LBUM), University of Montreal Hospital, 2099 Pavilion J. A. de Sève, \\ Montréal, QC, Canada H2L 2W5 \\ ${ }^{2}$ Centre de Recherche et d'Applications en Traitement de l'Image et du Signal (CREATIS), CNRS UMR 5515, Inserm U 630, \\ INSA de Lyon, 7 Avenue Jean Capelle, 69621 Villeurbanne Cedex, France
}

Received 20 April 2006; Revised 20 September 2006; Accepted 20 September 2006

Recommended by Tan Lee

A new formulation for the estimation of the time-scaling factor between two ultrasound signals is presented. The estimator is derived under the assumptions of a small time-scaling factor and signals with constant spectrum over its bandwidth. Under these conditions, we show that the proposed approach leads to a simple analytic formulation of the time-scaling factor estimator. The influences of an increase of the time-scaling factor and of signal-to-noise ratio (SNR) are studied. The mathematical developments of the expected mean and bias of the estimator are presented. An iterative version is also proposed to reduce the bias. The variance is calculated and compared to the Cramer-Rao lower bound (CRLB). The estimator characteristics are measured on flat-spectra simulated signals and experimental ultrasound scanner signals and are compared to the theoretical mean and variance. Results show that the estimator is unbiased and that variance tends towards the CRLB for SNR higher than $20 \mathrm{~dB}$. This is in agreement with typical ultrasound signals used in the medical field, as shown on typical examples. Effects of the signal spectrum shape and of the bandwidth size are evaluated. Finally, the iterative version of the estimator improves the quality of the estimation for SNR between 0 and $20 \mathrm{~dB}$ as well as the time-scaling factor estimation validity range (up to $15 \%$ ).

Copyright (C) 2007 Jérémie Fromageau et al. This is an open access article distributed under the Creative Commons Attribution License, which permits unrestricted use, distribution, and reproduction in any medium, provided the original work is properly cited.

\section{INTRODUCTION}

The interest of the time-scale variation between two signals was shown in many applications. As an example, in radar or sonar systems, the Doppler shift, between the received and the transmitted signals, allows the estimation of the relative velocity of a target [1]. In medical imaging, the Doppler shift enables the estimation of blood flow velocity [2] or strain of soft biological tissues by comparing a pair of ultrasonic signals acquired before and after tissue compression $[3,4]$, the time-scaling factor then represents the tissue strain. In most applications, this time-scaling factor is considered small. For example, the target velocity is small compared to the wave speed. Similarly, in tissue strain estimation, the compression applied to the tissue is assumed to be of small magnitude. Typically for practical applications in medical ultrasonic imaging, the small size of the regions of interest and the high frame rate mean that the time stretch to estimate is usually less than 0.01 .
Few methods have been reported in the literature to estimate time-scaling factors or Doppler shifts. They are based on the location research of the ambiguity function maximum [1, 5-7], using other time-frequency representations as Wigner distribution [8], or using an adaptive process [9]. In this paper, a time-scaling factor estimator is dependent on of the ambiguity function with a constant delay. An analytic formulation of the estimator is derived, it has the advantage to allow a direct estimation of the time-scaling factor between two signals without tracking the maximum of the ambiguity function. A specific case is exemplified by signals whose spectra are constant over their bandwidth. Under this assumption, we propose a fast method to estimate small time-scaling factors. The estimation is deduced from the calculation of the ambiguity function for the unique doublet: the time delay and time-scaling factor are fixed to zero. This method is particularly well adapted for our application which aims at providing an image of the biological tissue deformation. The paper is organized as follows. In Section 2, 
the formulation of the estimator is derived and its theoretical performance, mean, and variance are calculated. The influence of the time-scaling factor amplitude is investigated. Then, an iterative version of the estimation is also given. Our variance estimator is compared to the Cramer-Rao lower bound. In Section 3, the performance on simulated signals is assessed and compared with theoretical results. The influence of the bandwidth, time-scaling factor amplitude, and the spectrum shape are discussed. We show that the iterative version of the estimator compensates the bias caused by these parameters. Finally performances are evaluated on experimental signals.

\section{TIME-SCALING FACTOR ESTIMATION}

\subsection{Estimation formulation}

Let $\tilde{r}(t)$ be an analytic signal as a function of time:

$$
\tilde{r}(t)=r(t)+j \check{r}(t)=A(t) e^{2 j \pi f_{0} t},
$$

where $r(t)$ is the real part of $\tilde{r}(t), \check{r}(t)=H\{r(t)\}$ is the imaginary part and the Hilbert transform of $r(t), f_{0}$ is the central frequency, and $A(t)$ is the complex envelope.

Consider $\widetilde{s}(t)$ a pure time-scaled version of the signal $\tilde{r}(t)$ :

$$
\widetilde{s}(t)=\widetilde{r}((1+\varepsilon) t),
$$

where $\varepsilon$ represents the varying part of the time-scaling factor and the quantity to be estimated. It will be called the time-scaling factor in the following. Note that this formulation implies that if there was a time delay, it would be previously compensated. In practice for medical applications, there is no time delay and the beginning of the signal represents the region stuck to the ultrasound probe. When the probe is pressed on a tissue, this region undergoes the same movement that the probe undergoes, the beginning of the signal corresponds to the same area and there is no delay between signals before and after compression.

Consider the complex function $R_{\widetilde{r} \widetilde{S}}(\vartheta)$ defined as

$$
\begin{aligned}
R_{\widetilde{r} \tilde{s}}(\vartheta) & =\int_{-\infty}^{\infty} \tilde{r}(t) \tilde{s}^{*}(t(1-\vartheta)) d t \\
& =2\left(R_{r s}(\vartheta)+j R_{\check{r} s}(\vartheta)\right),
\end{aligned}
$$

where the superscript $*$ denotes the complex conjugation and $\vartheta$ the time-scaling factor variable. This relation is obtained by resolving signals into their real and imaginary components while using the Hilbert transform properties [10]. Note that the function $R_{\widetilde{r}}(\vartheta)$ can be interpreted as an ambiguity function with a zero delay. It becomes maximal when $\widetilde{\mathcal{s}}(t(1-\vartheta))=\tilde{r}(t)$, that is to say, $\vartheta=\varepsilon /(1+\varepsilon)$. In that case, $R_{\widetilde{r} s}(\vartheta)$ is the energy of the signal $r(t)$, a real positive value, and the imaginary part $R_{\check{r} s}(\vartheta)$ is a nearly linear function with a zero crossing for this value of $\vartheta$. To derive the estimator, only the imaginary part is considered taking advantage of its nearly linear behavior and its zero crossing.

The proposed approach for the time-scaling factor estimation is based on the function $R_{\breve{r} s}(\vartheta)$ evaluated in the vicinity of $\vartheta=0,[11]$. Replacing $s(t)$ by $r(t)$ in (2), we can write

$$
R_{\check{r} s}(0)=\int_{-\infty}^{\infty} \check{r}(t) r(t+\varepsilon t) d t .
$$

Applying Parseval's relation [12], (4) becomes

$$
R_{\check{r} s}(0)=\int_{-\infty}^{\infty} \check{R}(f) \cdot \frac{1}{|1+\varepsilon|} R^{*}\left(\frac{f}{1+\varepsilon}\right) d f,
$$

where $R(f)$ and $\check{R}(f)$ are, respectively, the Fourier transform of $r(t)$ and $\check{r}(t)$, and are linked together by the following relation:

$$
\check{R}(f)=-j \operatorname{sign}(f) R(f)
$$

where $\operatorname{sign}(\cdot)$ represents the sign function. Then, it follows that

$$
\begin{aligned}
R_{\check{r} s}(0)= & \frac{-j}{|1+\varepsilon|} \int_{-\infty}^{\infty} \operatorname{sign}(f) \cdot R(f) \cdot R^{*}\left(\frac{f}{1+\varepsilon}\right) d f \\
= & \frac{j}{|1+\varepsilon|}\left(\int_{-\infty}^{0} R(f) \cdot R^{*}\left(\frac{f}{1+\varepsilon}\right) d f\right. \\
& \left.-\int_{0}^{\infty} R(f) \cdot R^{*}\left(\frac{f}{1+\varepsilon}\right) d f\right) .
\end{aligned}
$$

From here, some assumptions are made on signal characteristics to derive the estimator. They are summarized as follows.

Hypothesis 1. Signals are assumed to have a constant spectrum over the bandwidth $B$. This assumption is the most restrictive but allows a simple expression of the estimator and gives a good approximation of the time-scaling factor for experimental signals even if they do not have such a spectrum, as it will be shown in Section 3.

Hypothesis 2. The applied time-scaling factor is considered to be very small, $\varepsilon \ll 1$, but can be negative or positive. Typically, one wishes is to estimate a time-scaling factor whose absolute value is around 0.01 . With this assumption, the estimator could be linearized using the Taylor expansion. Moreover, the absolute value $|1+\varepsilon|$ can be eliminated because $1+\varepsilon$ is always positive.

The first hypothesis implies that the signal energy is uniformly distributed over the bandwidth. Thus $R(f)$ is written:

$$
|R(f)|= \begin{cases}\sqrt{\frac{E_{r}}{2 B}} e^{-2 j \pi f t_{0}} & \text { if } f_{0}-\frac{B}{2} \leq|f| \leq f_{0}+\frac{B}{2} \\ 0 & \text { otherwise, }\end{cases}
$$

where the term $e^{-2 j \pi f t_{0}}$ is the phase term due to the group delay $t_{0}$ of the signal. For a time-limited signal between 0 and $T$, the group delay is chosen as the half-duration, $t_{0}=T / 2$. 
With this form of Fourier transform, (7) is modified as follows:

$$
\begin{aligned}
R_{\check{r} s}(0)= & \frac{j}{1+\varepsilon}\left(\int_{-\infty}^{0} R(f) \cdot R^{*}\left(\frac{f}{1+\varepsilon}\right) d f\right. \\
& \left.-\int_{0}^{\infty} R(f) \cdot R^{*}\left(\frac{f}{1+\varepsilon}\right) d f\right) \\
= & \frac{j \sqrt{E_{r} / 2 B}}{1+\varepsilon}\left(\int_{-f_{0}-B / 2}^{-f_{0}+B / 2} e^{-j \pi f T} \cdot R^{*}\left(\frac{f}{1+\varepsilon}\right) d f\right. \\
& \left.-\int_{f_{0}-B / 2}^{f_{0}+B / 2} e^{-j \pi f T} \cdot R^{*}\left(\frac{f}{1+\varepsilon}\right) d f\right) \\
= & \frac{j\left(E_{r} / 2 B\right)}{1+\varepsilon}\left(\int_{-f_{2}}^{-f_{1}} e^{-j \pi f T} e^{j \pi f T /(1+\varepsilon)} d f\right. \\
& \left.-\int_{f_{1}}^{f_{2}} e^{-j \pi f T} e^{j \pi f T /(1+\varepsilon)} d f\right),
\end{aligned}
$$

where, according to the $\varepsilon$ sign,

$$
\begin{aligned}
& f_{1}=\max \left(f_{0}-\frac{B}{2}, \frac{f_{0}-B / 2}{1+\varepsilon}\right), \\
& f_{2}=\min \left(\frac{f_{0}+B / 2}{1+\varepsilon}, f_{0}+\frac{B}{2}\right) .
\end{aligned}
$$

Because the signal $s(t)$ is a time-scaled version of $r(t)$, its Fourier transform undergoes a frequency shift and the bandwidth boundaries $f_{1}$ and $f_{2}$ change. Noting the energy of the signal $s(t)$, defined by $E_{s}=E_{r} /(1+\varepsilon)$, and using small timescaling factor assumption, (9) becomes

$$
\begin{aligned}
R_{\check{r} s}(0) & =\frac{j E_{s}}{2 B}\left(\int_{-f_{2}}^{-f_{1}} e^{-j \pi \varepsilon f T} d f-\int_{f_{1}}^{f_{2}} e^{-j \pi \varepsilon f T} d f\right) \\
& =\frac{-E_{s}}{2 \pi \varepsilon B T}\left(\left[e^{-j \pi \varepsilon f T}\right]_{-f_{2}}^{-f_{1}}-\left[e^{-j \pi \varepsilon f T}\right]_{f_{1}}^{f_{2}}\right) \\
& =\frac{2 E_{s}}{\pi \varepsilon B T}\left(\sin \left(\frac{\pi \varepsilon\left(f_{2}-f_{1}\right) T}{2}\right) \cdot \sin \left(\frac{\pi \varepsilon\left(f_{1}+f_{2}\right) T}{2}\right)\right) .
\end{aligned}
$$

According to the sign of $\varepsilon$ and to the possible values of $f_{1}$ and $f_{2},(9)$ can be written in a general way as

$$
\begin{aligned}
R_{\check{r} s}(0)= & \frac{2 E_{s}}{\pi \varepsilon B T} \cdot \sin \left(\frac{\pi T \varepsilon\left((2+\varepsilon) B / 2-|\varepsilon| f_{0}\right)}{2(1+\varepsilon)}\right) \\
& \cdot \sin \left(\frac{\pi T \varepsilon\left((2+\varepsilon) f_{0}-|\varepsilon| B / 2\right)}{2(1+\varepsilon)}\right) .
\end{aligned}
$$

With the second assumption that $\varepsilon \ll 1$, and after possible simplifications of negligible second-order terms in $\varepsilon$, the relation is simplified as

$$
\begin{aligned}
R_{\check{r s}}(0) & \approx \frac{2 E_{s}}{\pi \varepsilon B T} \cdot \sin \left(\frac{\pi \varepsilon B T}{2}\right) \cdot \sin \left(\pi f_{0} \varepsilon T\right) \\
& \approx E_{s} \operatorname{sinc}\left(\frac{\pi \varepsilon B T}{2}\right) \cdot \sin \left(\pi f_{0} \varepsilon T\right) .
\end{aligned}
$$

With the previous assumptions, this relation can be simplified. As the half bandwidth is smaller than the central frequency, $(B / 2) \varepsilon T$ tends towards zero faster than the term $f_{0} \varepsilon T$, the sinus function in the term $\sin (\pi(B / 2) \varepsilon T)$ is expanded to the first-order term. $R_{\check{r} s}(0)$ is expressed by

$$
R_{\check{r} s}(0) \approx E_{s} \sin \left(\pi f_{0} \varepsilon T\right) .
$$

The approximation resulting from the truncation of higher-order terms of the Taylor expansion is the most restrictive one, but it makes it possible to find a simple inverse relation with $\varepsilon$. This assumption will lead to a more effective estimation for small bandwidth signals. The expression of the time-scaling factor estimator is then deduced,

$$
\widehat{\varepsilon}=\frac{1}{\pi f_{0} T} \arcsin \left(\frac{R_{\check{r}}(0)}{E_{s}}\right) .
$$

Since $E_{s}$ and $R_{\check{r} s}(0)$ are computed from the signals $r(t)$ and $s(t)$, the time-scaling factor can be estimated directly from these two signals. In practice this estimator was dedicated to discrete signals, but the derivation was calculated with continuous signals for clarity of mathematical expressions. Discrete estimator formulation is derived from (15),

$$
\widehat{\varepsilon}=\frac{1}{\pi f_{0} N} \arcsin \left(\frac{\sum_{n=1}^{N} \check{r}[n] \cdot s[n]}{\sum_{n=1}^{N} s[n] \cdot s[n]}\right) .
$$

Performance of this initial estimator needs to be assessed when signals are corrupted with noise. Preliminary investigations deal with the estimation of any possible bias, which requires knowing the mean of the time-scaling factor estimate.

\subsection{Behavior of the estimator}

\subsubsection{Mean of the estimate}

Assume that noisy signals $x_{1}(t)$ and $x_{2}(t)$ are modeled by $r(t)$ and $s(t)$ corrupted by independent additive time-limited and Gaussian noise with zero mean and variance $\sigma^{2}$. Furthermore, the noises are stationary and ergodic. Their respective expressions are

$$
\begin{aligned}
& x_{1}(t)=r(t)+n_{1}(t), \\
& x_{2}(t)=s(t)+n_{2}(t)
\end{aligned}
$$

with

$$
\begin{gathered}
E\left[n_{i}(t)\right]=0, \\
E\left[n_{i}(t) n_{j}\left(t^{\prime}\right)\right]=\sigma^{2} \delta_{i j} \delta\left(t-t^{\prime}\right), \quad \delta_{i j} \text { the Kronecker delta, } \\
E\left[\check{n}_{i}(t) n_{j}\left(t^{\prime}\right)\right]=0, \quad i, j=1,2 .
\end{gathered}
$$


Note that for this problem, the delay is always 0 , no time shift is performed between the signals, and $t=t^{\prime}$ for the whole derivation. The time-scaling factor estimation is made between the signals $x_{1}(t)$ and $x_{2}(t)$, and from (15) becomes

$$
\begin{aligned}
\widehat{\varepsilon} & =\frac{1}{\pi f_{0} T} \arcsin \left(\frac{R_{\check{x}_{1} x_{2}}(0)}{E_{x_{2}}}\right) \\
& =\frac{1}{\pi f_{0} T} \arcsin \left(\frac{\int\left(\check{r}(t)+\check{n}_{1}(t)\right)\left(s(t)+n_{2}(t)\right) d t}{\int\left(s(t)+n_{2}(t)\right)\left(s(t)+n_{2}(t)\right) d t}\right) .
\end{aligned}
$$

The mean of the estimate is calculated in Appendix A. The result of the mean calculation can be expressed as a function of the signal-to-noise ratio (SNR),

$$
E[\widehat{\varepsilon}]=\frac{1}{\pi f_{0} T} \arcsin \left(\frac{\mathrm{SNR}}{\mathrm{SNR}+1} \operatorname{sinc}\left(\frac{\pi B \varepsilon T}{2}\right) \sin \left(\pi f_{0} \varepsilon T\right)\right),
$$

where $\mathrm{SNR}=E_{s} / T \sigma^{2}$ is the ratio between the signal energy and that of the noise during the observation time $T$. Thanks to the previous assumptions made to establish (13), that is, $|\varepsilon \ll 1|$, this relation can be expanded to give

$$
E[\widehat{\varepsilon}] \approx \frac{\varepsilon \mathrm{SNR}}{\mathrm{SNR}+1} .
$$

This relation is valid for any SNR. The estimator is unbiased for a high SNR. However, for a low SNR, a bias is present and the mean of the estimate tends towards the zero-mean of the noise,

$$
\begin{gathered}
\lim _{\mathrm{SNR} \rightarrow \infty} E[\hat{\mathcal{\varepsilon}}]=E\left[\hat{\mathcal{E}}_{\infty}\right]=\varepsilon, \\
\lim _{\mathrm{SNR} \rightarrow 0} E[\hat{\varepsilon}]=0 .
\end{gathered}
$$

\subsubsection{Variance}

To continue with the estimator characterization, the theoretical variance is estimated,

$$
\operatorname{var}(\widehat{\mathcal{\varepsilon}})=E\left[(\widehat{\varepsilon}-E[\widehat{\varepsilon}])^{2}\right]=E\left[\widehat{\mathcal{\varepsilon}}^{2}\right]-E[\widehat{\mathcal{\varepsilon}}]^{2} .
$$

The mean of the estimator $E[\widehat{\varepsilon}]$ was previously calculated. The calculation of the variance is developed in Appendix B. The result is

$$
\begin{aligned}
\operatorname{var}(\hat{\mathcal{\varepsilon}})=( & ((2+\varepsilon) \mathrm{SNR}+1) \cdot(\mathrm{SNR}+1)^{2} \\
& \left.+4 \mathrm{SNR}^{3} \operatorname{sinc}^{2}(\pi \varepsilon B T / 2) \cdot \sin ^{2}\left(\pi f_{0} \varepsilon T\right)\right) \\
/ & \left(T ( \pi f _ { 0 } T ) ^ { 2 } ( \mathrm { SNR } + 1 ) ^ { 2 } \left((\mathrm{SNR}+1)^{2}\right.\right. \\
& \left.\left.-\mathrm{SNR}^{2} \operatorname{sinc}^{2}(\pi \varepsilon B T / 2) \cdot \sin ^{2}\left(\pi f_{0} \varepsilon T\right)\right)\right) .
\end{aligned}
$$

Note that although it does not appear explicitly, variance has no dimension. Indeed, in expression (24), some constants in the numerator and the denominator come from a
Dirac integration $\int_{0}^{T} \delta\left(t-t^{\prime}\right) d t=1$, and have a time dimension (see Appendix B).

The expression of the variance is complex but its limits can easily be determined,

$$
\begin{gathered}
\lim _{\mathrm{SNR} \rightarrow \infty} \operatorname{var}(\widehat{\mathcal{\varepsilon}})=0, \\
\lim _{\mathrm{SNR} \rightarrow 0} \operatorname{var}(\widehat{\varepsilon})=\frac{1}{T\left(\pi f_{0} T\right)^{2}} .
\end{gathered}
$$

For a high SNR, the estimator variance tends towards zero. Noise becomes very small compared to the signal amplitude, and the estimation becomes perfectly deterministic. For a low SNR, the variance tends towards a constant value that depends on the signal length $T$. Signals are completely dominated by noise, and variance is bounded by the a priori parameter domain. This behavior was discussed for the timedelay estimation by Weiss and Weinstein [13], especially the fact that for a low SNR, the Cramer-Rao bound is not a satisfactory lower bound.

\subsubsection{Cramer-Rao bound}

The Cramer-Rao lower bound is the common reference to characterize estimation variance $[14,15]$. The problem becomes to calculate the Cramer-Rao lower bound for a Gaussian variable of which mean and standard deviation have been calculated in the previous section:

$$
\begin{aligned}
E\left[R_{\check{x}_{1} x_{2}}(0)\right] & =R_{\check{r} s}(0), \\
E\left[R_{\check{x}_{1} x_{2}}(0)^{2}\right]-E\left[R_{\check{x}_{1} x_{2}}(0)\right]^{2} & =\sigma_{R}^{2}=(2+\varepsilon) E_{s} \sigma^{2}+T \sigma^{4} .
\end{aligned}
$$

This is a well-known problem to calculate the CramerRao bound for a Gaussian variable. This bound is given by the inverse of the Fisher information matrix $\operatorname{var}(\widehat{\varepsilon}) \geq J^{-1}$ and is defined as

$$
J=-E\left\{\frac{\partial^{2} \ln p\left(R_{\check{x}_{1} x_{2}}(0) \mid \varepsilon\right)}{\partial \varepsilon^{2}}\right\}
$$

where $p\left(R_{\check{x}_{1} x_{2}}(0) \mid \varepsilon\right)$ is the conditional probability density function $R_{\check{x}_{1} x_{2}}(0)$. The log-likelihood function is

$$
\ln p\left(R_{\check{x}_{1} x_{2}}(0) \mid \varepsilon\right)=-\frac{1}{2} \ln \left(2 \pi \sigma_{R}^{2}-\frac{\left(R_{\check{x}_{1} x_{2}}(0)-R_{\check{r} s}(0)\right)^{2}}{2 \sigma_{R}^{2}}\right)
$$

The detail of the CRB calculation is given in Appendix C. Resolving (27) with this probability density function, the CRB calculation leads to

$$
\operatorname{var}(\widehat{\varepsilon}) \geq \frac{[(2+\varepsilon) \mathrm{SNR}+1]^{2}}{\operatorname{TSNR}^{2}[(2+\varepsilon) \mathrm{SNR}+1] K^{2}-\mathrm{SNR}^{2} / 2}
$$


with

$$
\begin{aligned}
K= & \frac{2}{\pi \varepsilon^{2} B T} \sin \left(\frac{\pi \varepsilon B T}{2}\right) \sin \left(\pi \varepsilon f_{0} T\right) \\
& -\frac{B+2 f_{0}}{2 B \varepsilon} \sin \left(\pi \varepsilon\left(\frac{B}{2}+f_{0}\right) T\right) \\
& +\frac{B-2 f_{0}}{2 B \varepsilon} \sin \left(\pi \varepsilon\left(\frac{B}{2}-f_{0}\right) T\right) .
\end{aligned}
$$

This bound serves as a reference to assess the estimator performances. The estimator is efficient if its variance tends towards the CRLB.

\subsubsection{Bias of the estimate}

The bias is calculated as the expression $b(\varepsilon)=E[\hat{\varepsilon}]-\varepsilon$, the difference between the mean time-scaling factor estimated between signals and the true one. This difference is mainly due to the Taylor expansion performed in (14). To assess bias value, (14) is inserted in (20), and the $R_{\widetilde{r s}}\left(\varepsilon_{0}\right)$ function is expanded in a Taylor series up to the third-order term. This order is chosen to be higher than the truncation order used in the estimator derivation (15) for a better precision:

$E[\hat{\varepsilon}]$

$$
\begin{aligned}
= & \frac{1}{\pi f_{0} T} \arcsin \left(\frac{\mathrm{SNR}}{\mathrm{SNR}+1} \operatorname{sinc}\left(\frac{\pi B \varepsilon T}{2}\right) \sin \left(\pi f_{0} \varepsilon T\right)\right) \\
\approx & \frac{1}{\pi f_{0} T} \arcsin \left(\frac{\mathrm{SNR}}{\mathrm{SNR}+1}\right. \\
& \left.\cdot \frac{2\left(\left(\pi f_{0} \varepsilon T\right)-\left(\pi f_{0} \varepsilon T\right)^{3} / 6\right)\left(\pi B \varepsilon T / 2-(\pi B \varepsilon T)^{3} /\left(2^{3} \times 6\right)\right)}{\pi B \varepsilon T}\right)
\end{aligned}
$$$$
\approx \frac{\mathrm{SNR}}{\pi f_{0} T(\mathrm{SNR}+1)}\left(\pi f_{0} T \varepsilon-f_{0} \frac{f_{0}^{2}+B^{2} / 4}{6}(\pi T \varepsilon)^{3}\right.
$$$$
\left.+\frac{1}{6}\left(\pi f_{0} T \varepsilon-f_{0} \frac{f_{0}^{2}+B^{2} / 4}{6}(\pi T \varepsilon)^{3}\right)^{3}\right)
$$$$
\approx \varepsilon-\frac{\varepsilon}{\mathrm{SNR}+1}-\frac{\mathrm{SNR}}{\mathrm{SNR}+1} \cdot \frac{B^{2}}{24}(\pi T)^{2} \varepsilon^{3} .
$$

Thus the bias is given by

$$
b(\varepsilon)=-\frac{\varepsilon}{\mathrm{SNR}+1}-\frac{\mathrm{SNR}}{\mathrm{SNR}+1} \cdot \frac{B^{2}}{24}(\pi T)^{2} \varepsilon^{3} .
$$

This proves that a bias remains with this estimator. An unknown error occurs, because it depends on the unknown parameter $\varepsilon$. SNR and bandwidth also have an important influence on the bias. If signals are narrowband, the error can be negligible. For example for a bandwidth of $10 \%$ of $f_{0}$, a time-scaling factor of 0.005 is estimated with a $0.1 \%$ bias at $30 \mathrm{~dB}$ SNR and with a $1 \%$ bias at $20 \mathrm{~dB}$ SNR. For more general cases, the bias can become very high. A good technique to improve the estimator characteristics in terms of SNR and bandwidth dependency is to use an iterative process.

\subsubsection{Iterative formulation}

An iterative formulation of the estimator is proposed to examine improvement in terms of bias compensation. In the iterative process, the time-scaling factor is estimated firstly, resulting in an estimated value $\hat{\varepsilon_{1}}$. Then the ambiguity function (3) is calculated in the vicinity of $\vartheta=\varepsilon_{1}$ to estimate the next iteration, and so on. The iterative formulation is then written as

$$
\widehat{\varepsilon}_{i+1}=\widehat{\varepsilon}_{i}+\frac{1}{\pi f_{0} T} \arcsin \left(\frac{R_{\check{r} s}\left(\widehat{\varepsilon}_{i}\right)}{E_{s}}\right),
$$

where $\widehat{\varepsilon}_{i}$ is the ith iteration and $\hat{\varepsilon}_{0}=0$. The effect of the iterative process will be discussed in Section 3 .

\section{RESULTS}

The estimation is first assessed on simulated signals. In practice, the estimator is dedicated to discrete signals; all estimated results are then calculated with the discrete version of the estimator described in (16). Two numerical signals are synthesized, as described in (1) and (2). Furthermore flat spectra are assigned to the signals to be coherent with the assumption made for the derivation. Their parameters are in their normalized form, and thus are dimensionless. The reference signal is defined from its Fourier transform as in (8):

$$
|R(f)|= \begin{cases}1 & \text { if } f_{0}-\frac{B}{2} \leq|f| \leq f_{0}+\frac{B}{2}, \\ 0 & \text { otherwise }\end{cases}
$$

where $f_{0}$ is the central frequency and $B$ is the bandwidth. With the models of signals and (34), discrete simulated signals are written

$$
\begin{aligned}
& r[n]=\operatorname{ifft}(R(f)), \\
& s[n]=r[(1+\varepsilon) n],
\end{aligned}
$$

where signals $r[n]$ and $s[n]$ differ by a pure time-scaling factor $\varepsilon=-0.005 . s[n]$ is calculated from $r[n]$ using a lowpass interpolation. In practice, real signal $r[n]$ is acquired and its Fourier transform calculated. In the Fourier representation, (6) is used. Then the inverse Fourier transform is applied to obtain the imaginary part of the signal, $\check{r}[n]$. Parameters are the signal length $N=2000$, and the sampling period $F_{s}=1$. The central frequency is $f_{0}=2 \cdot 10^{-2}$, the bandwidth of the signal is $B \approx 1.5 \cdot 10^{-2}$, and the fractional bandwidth is $B / f_{0} \approx 0.77$. Additive Gaussian and uncorrelated noises are added to $r[n]$ and $s[n]$. The SNR varies over -20 to $50 \mathrm{~dB}$. In order to perform a statistical study, a set of 1000 independent signals is simulated for each SNR, and mean and variance of the estimation result are calculated.

Figure 1 compares theoretical and simulated means of the time-scaling factor estimates as a function of the SNR. The time-scaling factor estimation was performed with a single iteration. The theoretical mean is calculated using the continuous-time equation (20). Note that the singleiteration estimate curve is very close to the theoretical one for 


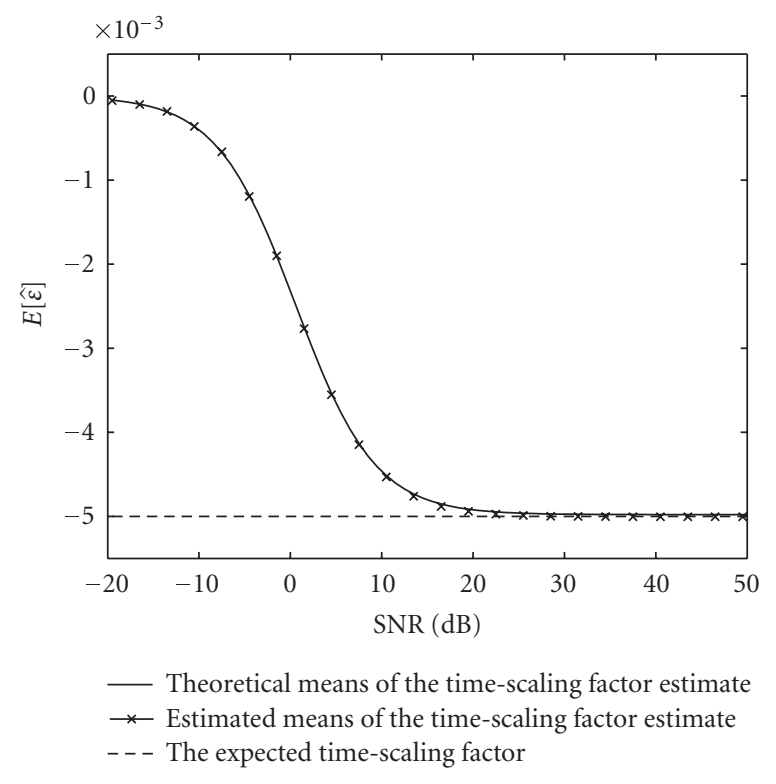

FIgURE 1: Theoretical and estimated means of the time-scaling factor estimate. The expected time-scaling factor to estimate is plotted for each SNR. Estimated means are calculated with 1 iteration. The estimated mean is calculated on a set of 1000 independent signals with a real time-scaling factor of $-5 \cdot 10^{-3}$.

any SNR, which proves that the estimator has similar characteristics in discrete-time representation and in continuoustime representation. The numerical values show that the algorithm estimator makes it possible to find the expected time-scaling factor, -0.005 for this case, with a relative error smaller than $1 \%$ when the SNR is greater than $20 \mathrm{~dB}$. Note that in medical applications, it is very common to deal with signals with an SNR higher than $20 \mathrm{~dB}$.

In Figure 2, similar curves are plotted for the theoreti$\mathrm{cal}$ and the estimated variances. Theoretical variance is calculated using (24). Once again, the similar behavior between continuous-time algorithm and discrete-time one can be pointed out. The Cramer-Rao bound is also plotted as a reference. For SNR higher to $12 \mathrm{~dB}$, Figure 2 proves that the estimator is efficient and achieves the same performances that the CRLB performs. Note that for low SNR, due to the fact that the estimation becomes biased in this region, estimator variance is smaller than the CRLB. For SNR smaller to $0 \mathrm{~dB}$, the variance tends towards a constant value related to the signal length (25). This corresponds to the variance of a uniformly distributed random variable. Such a performance level can always be achieved, regardless of signal observations, and is found when signals are completely dominated by noise.

With the iterative version of the estimator, the case of signals with a $10 \mathrm{~dB}$ SNR and an increasing time-scaling factor is plotted in Figure 3. With this mild conditions, after 2 iterations, the difference between the estimated time-scaling factor and the expected one is not easy to discern. The relative error for a time-scaling factor to estimate at 0.02 is smaller than $10^{-6} \%$. For higher time-scaling factors, more iterations

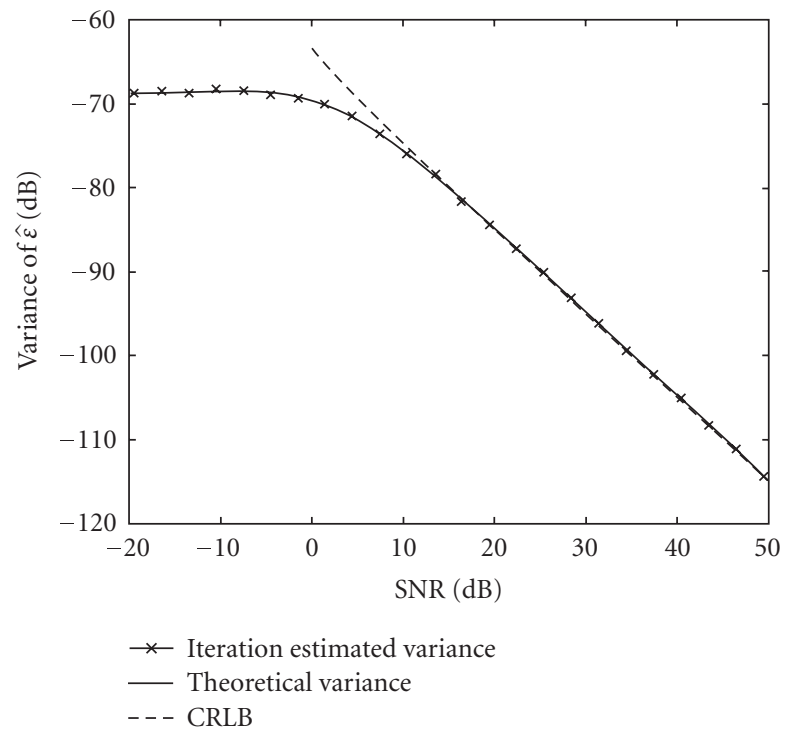

FIgURE 2: One iteration estimated variance of the time-scaling factor estimation according to the SNR, it is compared to theoretical variance and CRLB. The estimated variance is calculated on a set of 1000 independent signals with an applied time-scaling factor of $-5 \cdot 10^{-3}$.

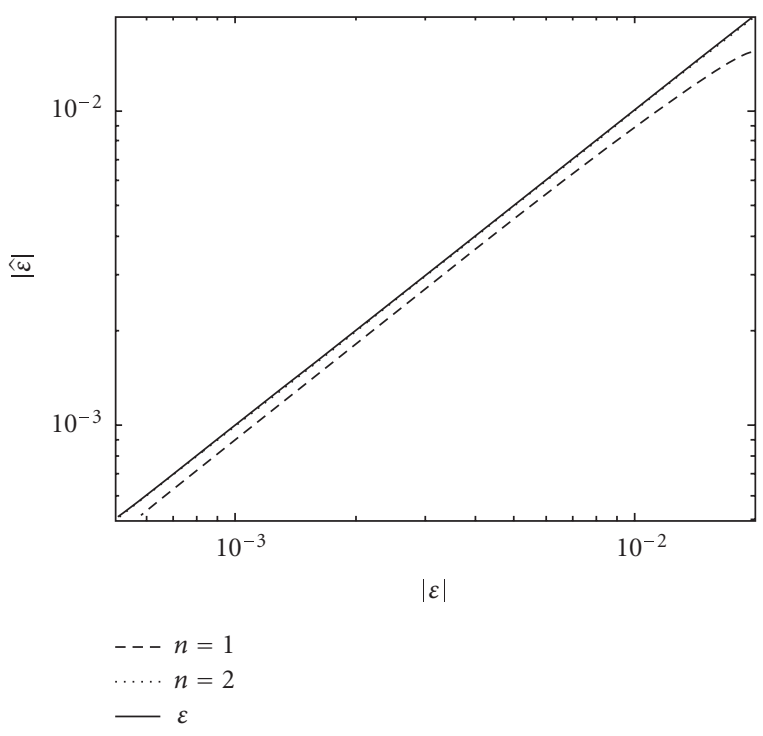

FIgURE 3: Comparison of the estimated time-scaling factor for $n=$ 1 iteration, $n=2$ iterations, and the true time-scaling factor $\varepsilon$ as a function of the applied time-scaling factor. Simulated signals have a flat spectrum with $B / f_{0} \approx 0.77$ and a $10 \mathrm{~dB}$ SNR.

may be required. For example, with similar signals, a timescaling factor of 0.1 is estimated with a relative error smaller than $10^{-6} \%$ after 16 iterations.

On a second stage, the estimator is assessed on simulated signals with a Gaussian spectrum to visualize the influence of flat-spectrum hypothesis in the mathematical derivation. 


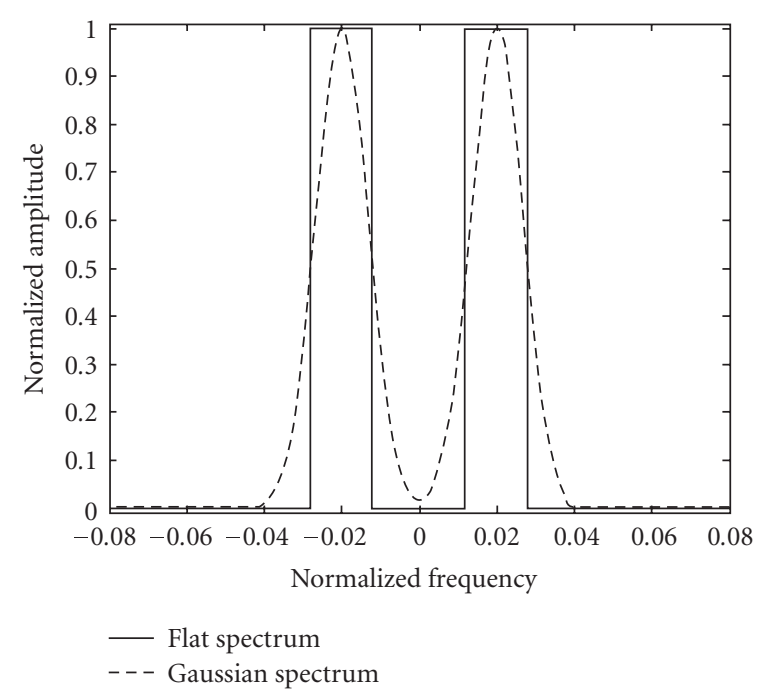

(a)

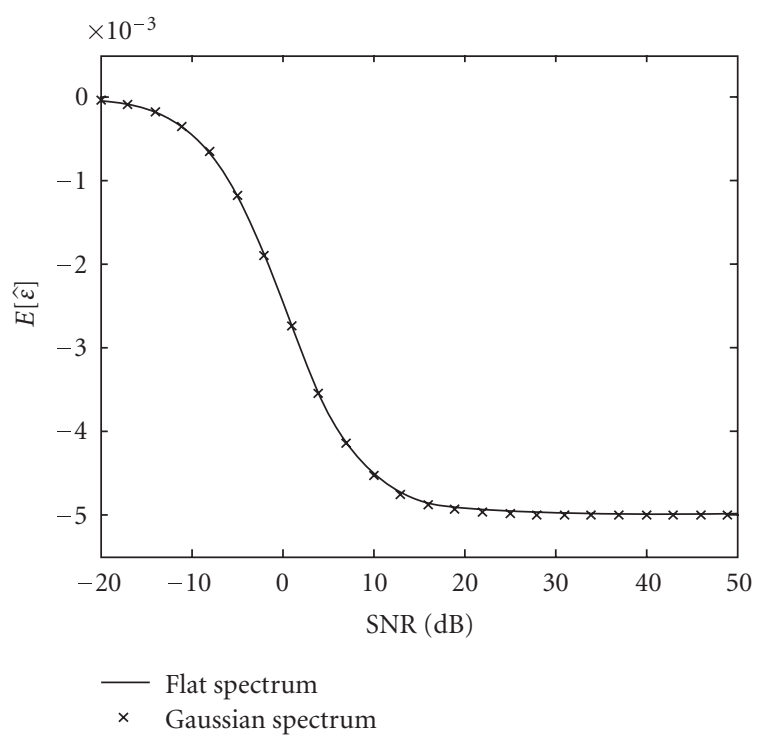

(b)

FIGURE 4: Influence of the spectrum shape on estimation. (a) Fourier transform of signals with a flat spectrum and Gaussian spectrum. (b) Mean time-scaling factor estimated from signals with a flat spectrum and from signal with a Gaussian spectrum. Except the shape, all spectrum characteristics (central frequency, bandwidth) are similar. The shape change does not make difference on the estimated timescaling factor.

Signal parameters are identical to the previous example, especially, central frequency and bandwidth are kept to $f_{0}=$ $2 \cdot 10^{-2}$ and $B \approx 1.5 \cdot 10^{-2}$, although the spectra shape changes, see Figure 4(a). The estimation with such a simulation shows no significant difference with the previous case of flat-spectrum signals, see Figure 4(b). Mean time-scaling factor calculated with Gaussian spectrum signals fits to the one obtained with flat spectrum signals. This means that although Hypothesis 1 may appear restrictive, it does not affect the estimation.

As mentioned previously, the most restrictive assumption was performed between (13) and (14), to have a simple inverse relation between $\varepsilon$ and $R_{\widehat{r} s}(0)$. For this purpose, the bandwidth-dependent term is considered small compared to the $f_{0}$ dependent term. This assumption is true for narrowband signals, but is not as well valid when $B$ is of the same order as that of $f_{0}$. The influence of the bandwidth size is represented on Figure 5 where the estimation relative error is plotted as a function of the ratio $B / f_{0}$. A theoretical relative error was calculated by including the nonsimplified theoretical version (20) with an SNR of $50 \mathrm{~dB}$ to avoid bias due to the noise. As expected, error increases with higher bandwidth size, leading to an error of $6 \%$ for the limit case when $B=1.8 f_{0}$. In practice, ultrasound signals we used have a bandwidth ratio of about 0.7 . The estimated bias is also calculated on similar signals for one and two iterations. Figure 5 shows that the single iteration estimation has a behavior similar to the theory with an error increasing with bandwidth size, however the iterative estimation version compensates this error.

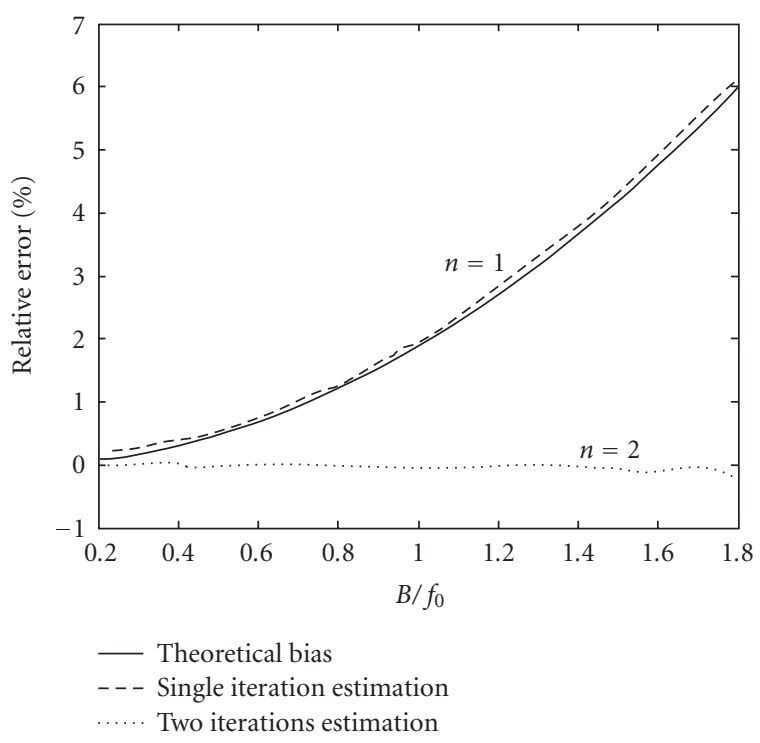

FIGURE 5: Influence of the bandwidth size. Estimation relative error percentage as a function of the ratio $B / f_{0}$. Theoretical bias, single iteration estimation, and two iterations estimation are plotted. With only one iteration, a large bandwidth can jeopardize the estimation, but the iterative estimator version can compensate this error.

Finally, the estimator is applied to typical clinically acquired ultrasound signals. The central frequency of these signals was $f_{0}=5.6 \mathrm{MHz}$, the sampling frequency was $F_{s}=250 \mathrm{MHz}$, and the SNR was measured at about $20 \mathrm{~dB}$. 


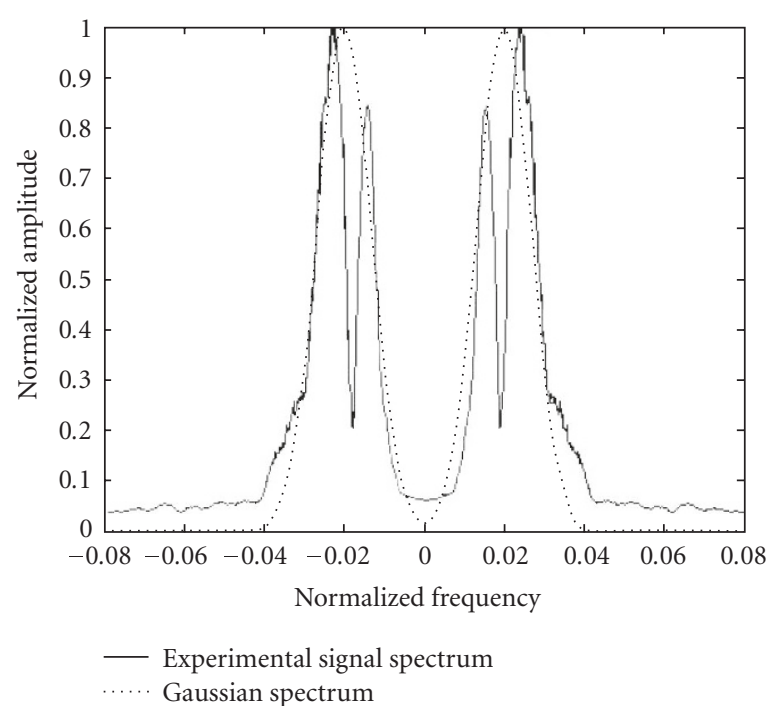

(a)

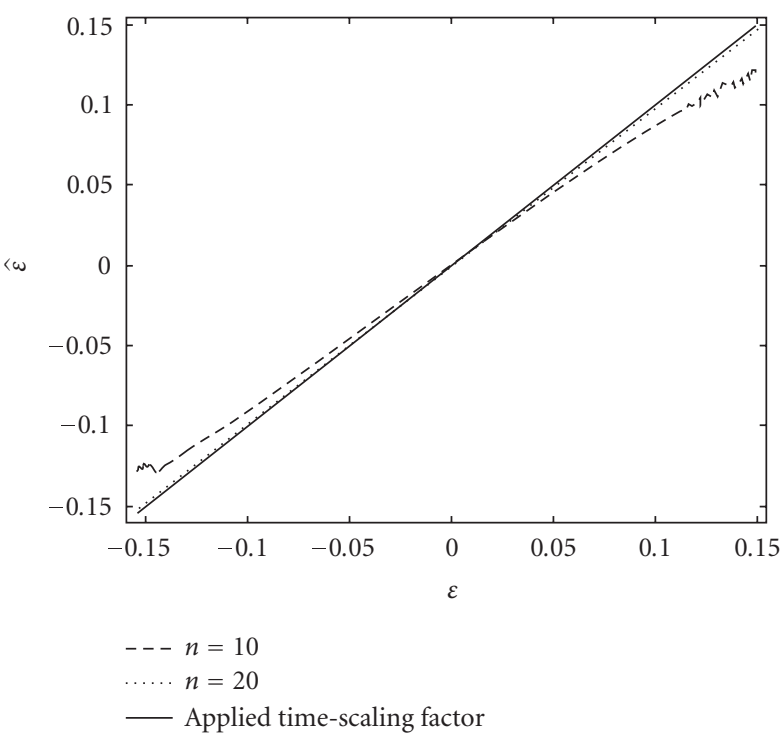

(b)

FIGURE 6: (a) Experimental signal spectrum; for comparison, the Gaussian spectra used in simulation is plotted behind. (b) Time-scaling factor estimated from ultrasound scanner signals. The second signal was artificially stretched from $-15 \%$ to $15 \%$. The time-scaling factors were calculated with $n=10$ iterations and $n=20$ iterations. For comparison, the applied time-scaling factor is also plotted.

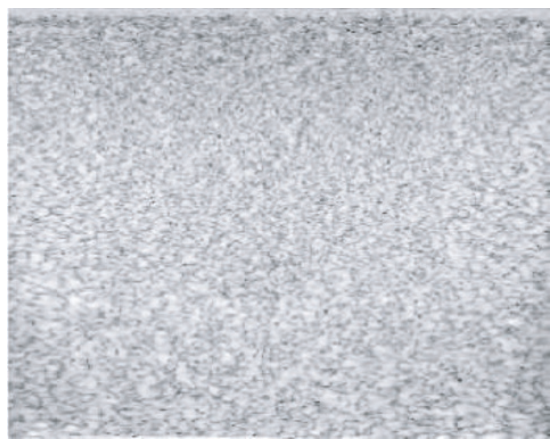

(a)

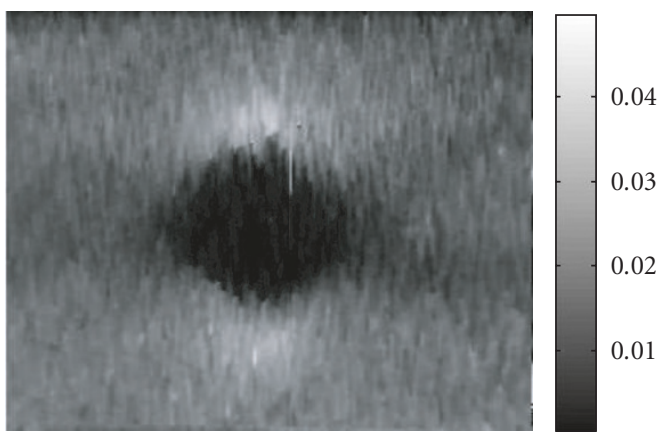

(b)

FIgURE 7: Application of the estimator on image of a sponge phantom within which is embedded a hard inclusion. (a) Ultrasound image of the phantom, (b) corresponding time-scaling factor distribution. The time-scaling factor in the inclusion is smaller, showing a harder elasticity in dark.

A signal spectrum is plotted with the Gaussian spectrum used for simulation, see Figure 6(a). They were voluntarily chosen similar in bandwidth and central frequency. Signals were artificially stretched from $-15 \%$ to $15 \%$, to cover a large range of scaling factors. For the high time-scaling factor to estimate, a high number of iterations can be necessary to converge through the expected mean value. Figure 6(b) shows the different estimates for $n=10$ iterations and $n=20$ iterations compared to the true time-scaling factor. It demonstrates the estimator ability to assess high timescaling factors, as long as the number of iterations is well adapted. In practice, this estimator is used to provide an image of tissue strain. Time-scaling factors are calculated on small windows of the whole signal. The calculation window is moved along the signal and the time-scaling factor that has been estimated on a window serves as an initial value for the estimation on the next window. Distance between two consecutive windows is small enough to have a small difference of the time-scaling factor estimated on each of them $(0.005$ is a typical value) and one iteration is generally sufficient. As an example, an ultrasound image and the corresponding timescaling factor distribution are plotted in Figure 7. In this case, the time-scaling factor describes the tissue deformation. The time-scaling factor distribution (Figure 7(b)) is obtained 
comparing two ultrasound images. One of the image was acquired for a rest state of the phantom (Figure 7(b)), and the second image was acquired after a slight compression. A dark spheric inclusion can be observed in the time-scaling factor map, whereas it is not visible on the ultrasound scanner image. The dark inclusion represents a region harder than the surrounding tissues because deformation in this area is smaller. Note that the whole image was calculated with a single iteration.

\section{CONCLUSION}

In this paper, we describe a simple method to estimate small time-scaling factors between two signals. The time-scaling factor estimation is given by an analytic expression, which provides a fast estimation. The function $R_{\widetilde{r}}\left(\varepsilon_{0}\right)$ is introduced, it can be considered as a complex ambiguity function with a zero-time delay. Our estimator uses only one value of the imaginary part of $R_{\widetilde{r} \tilde{S}}\left(\varepsilon_{0}\right)$, corresponding to the zerotime scale $\varepsilon_{0}=0$. The influence of SNR, applied time-scaling factor amplitude, bandwidth, and spectrum shape are studied and show how the bias increases depending on these parameters. A reduced bias version is proposed to improve the estimation using an iterative process. Theoretical characteristics of the estimator, mean, and variance are calculated and serve to assess estimator performance. The estimation variance is compared to the Cramer-Rao bound, and shows its efficiency. Application of the estimator on simulated signals with a single iteration demonstrates that mean and variance estimates are reliable for time-scaling factors smaller than 0.01 with an SNR higher than $20 \mathrm{~dB}$. These amplitudes may appear small but are typical of applications such as strain estimation in medical imaging. Furthermore, the iterative version allows for the estimation of time-scaling factor up to 0.15 , or on signals with smaller SNR.

\section{APPENDICES}

\section{A. MEAN OF THE ESTIMATE}

The theoretical mean of the estimator is developed from (19),

$$
\begin{aligned}
E[\hat{\varepsilon}] & =E\left[\frac{1}{\pi f_{0} T} \arcsin \left(\frac{R_{\check{x}_{1} x_{2}}(0)}{E_{x_{2}}}\right)\right] \\
& =\frac{1}{\pi f_{0} T} E\left[\arcsin \left(\frac{\int\left(\check{r}(t)+\check{n}_{1}(t)\right)\left(s(t)+n_{2}(t)\right) d t}{\int\left(s(t)+n_{2}(t)\right)\left(s(t)+n_{2}(t)\right) d t}\right)\right] .
\end{aligned}
$$

This relation is nonlinear; to go further, the process of the mean calculation is linearized. For this purpose, two random variables are introduced. The numerator is decomposed in a term with a zero variance (deterministic) and in a term with a zero mean,

$$
R_{\check{x}_{1} x_{2}}(0)=E\left[R_{\check{x}_{1} x_{2}}(0)\right]+\eta_{R},
$$

where $\eta_{R}$ is a zero-mean random variable with variance $\operatorname{Var}\left(\eta_{R}\right)=\operatorname{Var}\left(R_{\check{x}_{1} x_{2}}(0)\right)$. First, the deterministic part, termed
$E\left[R_{\check{x}_{1} x_{2}}(0)\right]$, is calculated:

$$
\begin{aligned}
E\left[R_{\check{x}_{1} x_{2}}(0)\right]= & E\left[\int\left(\check{r}(t)+\check{n}_{1}(t)\right)\left(s(t)+n_{2}(t)\right) d t\right] \\
= & \int E[\check{r}(t) s(t)] d t+\int E\left[\check{r}(t) n_{2}(t)\right] d t \\
& +\int E\left[\check{n}_{1}(t) s(t)\right] d t+\int E\left[\check{n}_{1}(t) n_{2}(t)\right] d t,
\end{aligned}
$$

where $r(t)$ and $s(t)$ are deterministic signals. Since noises are zero mean and circular (18), the mean of the numerator is

$$
E\left[R_{\check{x}_{1} x_{2}}(0)\right]=R_{\check{r} s}(0) .
$$

In the same way, a random variable is introduced in the denominator, which can be decomposed to

$$
E_{x_{2}}=E\left[E_{x_{2}}\right]+\eta_{E}
$$

where $\eta_{E}$ is a zero-mean random variable with variance $\operatorname{Var}\left(\eta_{E}\right)=\operatorname{Var}\left(E_{x_{2}}\right)$. Again the deterministic part $E_{x_{2}}$ is calculated,

$$
\begin{aligned}
E\left[E_{x_{2}}\right]= & E\left[\int\left(s(t)+n_{2}(t)\right)\left(s(t)+n_{2}(t)\right) d t\right] \\
= & \int s(t) s(t) d t+2 \int E\left[s(t) n_{2}(t)\right] d t \\
& +\int E\left[n_{2}(t) n_{2}(t)\right] d t \\
= & E_{s}+T \sigma^{2} .
\end{aligned}
$$

Thus, the estimator can be linearized near the point $E\left[R_{\check{x}_{1} x_{2}}(0)\right] / E\left[E_{x_{2}}\right]$ using the Taylor series expansion of the function arcsin,

$$
\begin{aligned}
\widehat{\varepsilon}= & \frac{1}{\pi f_{0} T} \arcsin \left(\frac{R_{\check{x}_{1} x_{2}}(0)}{E_{x_{2}}}\right) \\
= & \frac{1}{\pi f_{0} T} \arcsin \left(\frac{R_{\check{r} s}(0)}{E_{s}+T \sigma^{2}}\right)+\frac{\eta_{R}}{\pi f_{0} T \sqrt{E\left[E_{x_{2}}\right]^{2}-E\left[R_{\check{x}_{1} x_{2}}(0)\right]^{2}}} \\
& -\frac{\eta_{E} E\left[R_{\check{x}_{1} x_{2}}(0)\right]}{\pi f_{0} T E\left[E_{x_{2}}\right] \sqrt{E\left[E_{x_{2}}\right]^{2}-E\left[R_{\check{x}_{1} x_{2}}(0)\right]^{2}}} .
\end{aligned}
$$

As $\eta_{R}$ and $\eta_{E}$ are zero mean, the mean of the estimate $\hat{\varepsilon}$ is

$$
E[\hat{\mathcal{\varepsilon}}]=\frac{1}{\pi f_{0} T} \arcsin \left(\frac{R_{\check{r} s}(0)}{E_{s}+T \sigma^{2}}\right) .
$$

By inserting (13), and the definition of the SNR in (A.8), the mean of the time-scaling factor estimate can be expressed as a function of the SNR,

$$
E[\hat{\varepsilon}]=\frac{1}{\pi f_{0} T} \arcsin \left(\frac{\operatorname{sinc}(\pi B \varepsilon T / 2) \sin \left(\pi f_{0} \varepsilon T\right)}{1+1 / \mathrm{SNR}}\right) .
$$




\section{B. VARIANCE OF THE ESTIMATE}

To calculate the variance, the Taylor expansion used to linearize the estimator (A.7) is inserted to the definition of the variance. The variance is

$$
\begin{gathered}
E\left[(\hat{\varepsilon}-E[\hat{\varepsilon}])^{2}\right] \\
=\frac{1}{\left(\pi f_{0} T\right)^{2}} E\left[\left(\frac{\eta_{R}}{\sqrt{E\left[E_{x_{2}}\right]^{2}-E\left[R_{\check{x}_{1} x_{2}}(0)\right]^{2}}}\right.\right. \\
\left.\left.-\frac{\eta_{E} E\left[R_{\check{x}_{1} x_{2}}(0)\right]}{E\left[E_{x_{2}}\right] \sqrt{E\left[E_{x_{2}}\right]^{2}-E\left[R_{\check{x}_{1} x_{2}}(0)\right]^{2}}}\right)^{2}\right] \\
=\frac{1}{\left(\pi f_{0} T\right)^{2}} E\left[\left(\frac{\eta_{R} E\left[E_{x_{2}}\right]-\eta_{E} E\left[R_{\check{x}_{1} x_{2}}(0)\right]}{\left.E\left[E_{x_{2}}\right] \sqrt{E\left[E_{x_{2}}\right]^{2}-E\left[R_{\check{x}_{1} x_{2}}(0)\right]^{2}}\right)^{2}}\right]\right. \\
=\frac{1}{\left(\pi f_{0} T\right)^{2}} \frac{\operatorname{Var}\left(\eta_{R}\right) E\left[E_{x_{2}}\right]^{2}+\operatorname{Var}\left(\eta_{E}\right) E\left[R_{\check{x}_{1} x_{2}}(0)\right]^{2}}{E\left[E_{x_{2}}\right]^{2}\left(E\left[E_{x_{2}}\right]^{2}-E\left[R_{\check{x}_{1} x_{2}}(0)\right]^{2}\right)} .
\end{gathered}
$$

It is necessary to calculate $\operatorname{Var}\left(\eta_{R}\right)$ and $\operatorname{Var}\left(\eta_{E}\right)$. The secondorder moments of $R_{\check{x}_{1} x_{2}}^{2}(0)$ and $E_{x_{2}}$ are developed.

Firstly, the second-order moment of the numerator is calculated,

$$
\begin{aligned}
R_{\check{x}_{1} x_{2}}^{2}(0)= & \left(\int\left(\check{r}(t)+\check{n}_{1}(t)\right)\left(s(t)+n_{2}(t)\right) d t\right)^{2} \\
= & R_{\check{r} s}^{2}(0)+\iint\left(\check{r}(t) n_{2}(t) \check{r}\left(t^{\prime}\right) n_{2}\left(t^{\prime}\right)\right) d t d t^{\prime} \\
& +\iint\left(\check{n}_{1}(t) s(t) \check{n}_{1}\left(t^{\prime}\right) s\left(t^{\prime}\right)\right) d t d t^{\prime} \\
& +\iint\left(\check{n}_{1}(t) n_{2}(t) \check{n}_{1}\left(t^{\prime}\right) n_{2}\left(t^{\prime}\right)\right) d t d t^{\prime} \\
& +2 \iint\left(\check{r}(t) s(t) \check{r}^{\prime}\left(t^{\prime}\right) n_{2}\left(t^{\prime}\right)\right) d t d t^{\prime} \\
& +2 \iint\left(\check{r}(t) s(t) \check{n}_{1}\left(t^{\prime}\right) s\left(t^{\prime}\right)\right) d t d t^{\prime} \\
& +2 \iint\left(\check{r}(t) s(t) \check{n}_{1}\left(t^{\prime}\right) n_{2}\left(t^{\prime}\right)\right) d t d t^{\prime} \\
& +2 \iint\left(\check{r}(t) n_{2}(t) \check{n}_{1}\left(t^{\prime}\right) s\left(t^{\prime}\right)\right) d t d t^{\prime} \\
& +2 \iint\left(\check{r}(t) n_{2}(t) \check{n}_{1}\left(t^{\prime}\right) n_{2}\left(t^{\prime}\right)\right) d t d t^{\prime} \\
& +2 \int \check{n}_{1}(t) s(t) \check{n}_{1}\left(t^{\prime}\right) n_{2}\left(t^{\prime}\right) d t d t^{\prime},
\end{aligned}
$$

and its mean is the second-order moment. $\widetilde{r}(t)$ and $\widetilde{s}(t)$ are deterministic signals. All odd moments of the noise are zero. As signals have a finite-time length $T$, the integration over the time interval can be limited between 0 and $T$, and using properties of signals and noise,

$$
\begin{gathered}
\int_{0}^{T} \check{r}(t) \check{r}(t) d t=E_{r}=(1+\varepsilon) E_{s}, \\
\int_{0}^{T} s(t) s(t) d t=E_{s}, \\
\left.E\left[\int_{0}^{T} \check{r}(t) n_{i}(t) \check{r}\left(t^{\prime}\right) n_{j}\left(t^{\prime}\right)\right) d t d t^{\prime}\right] \\
=E_{r} \sigma^{2} \delta_{i j} \iint_{0}^{T} \delta\left(t-t^{\prime}\right) d t d t^{\prime}=T E_{r} \sigma^{2} \delta_{i j},
\end{gathered}
$$

where $\delta\left(t-t^{\prime}\right)$ is the delta function. Note that this last relation has the dimension of a square energy; a time component equal to $\int_{0}^{T} \delta\left(t-t^{\prime}\right) d t=1$ is present even if it does not appear explicitly. Finally, the second-order moment of the numerator is

$$
E\left[R_{\check{x}_{1} x_{2}}^{2}(0)\right]=R_{\check{r} s}^{2}(0)+(2+\varepsilon) E_{s} \sigma^{2}+T \sigma^{4} .
$$

Then $\operatorname{Var}\left(\eta_{R}\right)$ is

$$
\begin{aligned}
\operatorname{Var}\left(\eta_{R}\right) & =\operatorname{Var}\left(R_{\check{x}_{1} x_{2}}(0)\right) \\
& =E\left[R_{\check{x}_{1} x_{2}}^{2}(0)\right]-R_{\check{r} s}^{2}(0) \\
& =(2+\varepsilon) E_{s} \sigma^{2}+T \sigma^{4} .
\end{aligned}
$$

Secondly, a similar development is performed for the denominator,

$$
\begin{gathered}
E_{x_{2}}^{2}=\left(\int\left(s(t)+n_{2}(t)\right)\left(s(t)+n_{2}(t)\right) d t\right)^{2} \\
=\left[E_{s}^{2}+4 \iint s(t) n_{2}(t) s\left(t^{\prime}\right) n_{2}\left(t^{\prime}\right) d t d t^{\prime}\right. \\
+\iint n_{2}(t) n_{2}(t) n_{2}\left(t^{\prime}\right) n_{2}\left(t^{\prime}\right) d t d t^{\prime} \\
+4 \iint s(t) s(t) s\left(t^{\prime}\right) n_{2}\left(t^{\prime}\right) d t d t^{\prime} \\
+2 \iint s(t) s(t) n_{2}\left(t^{\prime}\right) n_{2}\left(t^{\prime}\right) d t d t^{\prime} \\
\left.+4 \iint s(t) n_{2}(t) n_{2}\left(t^{\prime}\right) n_{2}\left(t^{\prime}\right) d t d t^{\prime}\right] .
\end{gathered}
$$

Using signal and noise properties, (B.12), and

$$
\left.E\left[\int_{0}^{T} n_{i}(t) n_{j}(t)\right) d t\right]=T \sigma^{2} \delta_{i j}
$$

the second-order moment of $E_{x_{2}}$ can be written:

$$
\begin{aligned}
E\left[E_{x_{2}}^{2}\right] & =E_{s}^{2}+E_{s} \sigma^{2}(4+2 T)+T^{2} \sigma^{4} \\
& =\left(E_{s}+T \sigma^{2}\right)^{2}+4 E_{s} \sigma^{2} .
\end{aligned}
$$


Then $\operatorname{Var}\left(\eta_{E}\right)$ is

$$
\operatorname{Var}\left(\eta_{E}\right)=E\left[E_{x_{2}}^{2}\right]-\left(E_{s}+T \sigma^{2}\right)^{2}=4 E_{s} \sigma^{2}
$$

Obtaining the variance expression of $\eta_{R}$ and $\eta_{E}$ and the relation (B.10), the variance of the estimate is

$$
\operatorname{var}(\hat{\varepsilon})=\frac{\left((2+\varepsilon) E_{s} \sigma^{2}+T \sigma^{4}\right) \cdot\left(E_{s}+T \sigma^{2}\right)^{2}+4 E_{s} \sigma^{2} R_{\check{r} s}^{2}(0)}{\left(\pi f_{0} T\right)^{2}\left(E_{s}+T \sigma^{2}\right)^{2}\left(\left(E_{s}+T \sigma^{2}\right)^{2}-R_{\check{r} s}^{2}(0)\right)}
$$

Knowing the SNR definition and $R_{\check{r} s}(0)$ theoretical expression (13), the variance is finally

$$
\begin{aligned}
\operatorname{var}(\hat{\varepsilon})=( & ((2+\varepsilon) \mathrm{SNR}+1) \cdot(\mathrm{SNR}+1)^{2} \\
& \left.+4 \mathrm{SNR}^{3} \operatorname{sinc}^{2}(\pi \varepsilon B T / 2) \cdot \sin ^{2}\left(\pi f_{0} \varepsilon T\right)\right) \\
/( & T\left(\pi f_{0} T\right)^{2}(\mathrm{SNR}+1)^{2}\left((\mathrm{SNR}+1)^{2}\right. \\
& \left.\left.-\mathrm{SNR}^{2} \operatorname{sinc}^{2}(\pi \varepsilon B T / 2) \cdot \sin ^{2}\left(\pi f_{0} \varepsilon T\right)\right)\right) .
\end{aligned}
$$

\section{CRAMER-RAO BOUND}

Consider $R_{\check{x}_{1} x_{2}}(0)$ to be a Gaussian variable with its mean $E\left\{R_{\check{x_{1} x_{2}}}(0)\right\}=R_{\check{s} r}(0)$ and its variance $\operatorname{var}\left(R_{\check{x_{1} x_{2}}}(0)\right)=(2+$ ع) $E_{s} \sigma^{2}+T \sigma^{4}=\sigma_{R}^{2}$.

Its probability function is

$$
P\left(R_{\check{x}_{1} x_{2}}(0) \mid \varepsilon\right)=\frac{1}{\sqrt{2 \pi \sigma_{R}^{2}}} e^{-\left(R_{x_{1} x_{2}}(0)-R_{\check{s}}(0)\right)^{2} / 2 \sigma_{R}^{2}}
$$

The Cramer-Rao lower bound is given knowing the Fisher information matrix:

$$
J=-E\left\{\frac{\partial^{2} \ln p\left(R_{\check{x}_{1} x_{2}}(0) \mid \varepsilon\right)}{\partial \varepsilon^{2}}\right\}
$$

The density probability of the function $R_{\check{x}_{1} x_{2}}(0)$ being Gaussian, the log-likelihood function is

$$
\ln p\left(R_{\check{x}_{1} x_{2}}(0) \mid \varepsilon\right)=-\frac{1}{2} \ln \left(2 \pi \sigma_{R}^{2}\right)-\frac{\left(R_{\check{x}_{1} x_{2}}(0)-R_{\check{r} s}(0)\right)^{2}}{2 \sigma_{R}^{2}} .
$$

So the first and second derivates are

$$
\begin{aligned}
& \frac{\partial \ln p}{\partial \varepsilon} \\
& =-\frac{1}{\sigma_{R}} \frac{\partial \sigma_{R}}{\partial \varepsilon}+\sigma_{R}\left(\frac{\left(R_{\check{x}_{1} x_{2}}(0)-R_{\check{r} s}(0)\right)\left(\partial R_{\check{r} s}(0) / \partial \varepsilon\right)}{\sigma_{R}^{3}}\right. \\
& \left.+\frac{\left(\partial \sigma_{R} / \partial \varepsilon\right)\left(R_{\check{x}_{1} x_{2}}(0)-R_{\check{r} s}(0)\right)^{2}}{\sigma_{R}^{3}}\right) \\
& \frac{\partial^{2} \ln p}{\partial \varepsilon^{2}} \\
& =-\frac{\sigma_{R}\left(\partial^{2} \sigma_{R} / \partial \varepsilon^{2}\right)-\left(\partial \sigma_{R} / \partial \varepsilon\right)^{2}}{\sigma_{R}} \\
& +\sigma_{R}^{3}\left(\frac{\left(R_{\check{x}_{1} x_{2}}(0)-R_{\check{r} s}(0)\right)\left(\partial \sigma_{R} / \partial \varepsilon\right)\left(\partial R_{\check{r} s}(0) / \partial \varepsilon\right)}{\sigma_{R}^{6}}\right. \\
& -\frac{\sigma_{R}\left(\partial R_{\check{r} s}(0) / \partial \varepsilon\right)^{2}+\sigma_{R}\left(R_{\check{x}_{1} x_{2}}(0)-R_{\check{r} s}(0)\right) \partial^{2} R_{\check{r} s}(0) / \partial \varepsilon^{2}}{\sigma_{R}^{6}} \\
& +\frac{\left(\partial^{2} \sigma_{R} / \partial \varepsilon^{2}\right)\left(R_{\check{x}_{1} x_{2}}(0)-R_{\check{r} s}(0)\right)^{2}}{\sigma_{R}^{6}} \\
& \left.-\frac{2\left(\partial \sigma_{R} / \partial \varepsilon\right)\left(R_{\check{x}_{1} x_{2}}(0)-R_{\check{r} s}(0)\right) \partial R_{\check{r} s}(0) / \partial \varepsilon}{\sigma_{R}^{6}}\right) \\
& -3 \sigma_{R}^{2}\left(\partial \sigma_{R} / \partial \varepsilon\right)\left(\frac{\sigma_{R}\left(R_{\check{x}_{1} x_{2}}(0)-R_{\check{r} s}(0)\right)\left(\partial R_{\check{r} s}(0) / \partial \varepsilon\right)}{\sigma_{R}^{6}}\right. \\
& \left.+\frac{\left(\partial \sigma_{R} / \partial \varepsilon\right)\left(R_{\check{x}_{1} x_{2}}(0)-R_{\check{r} s}(0)\right)}{\sigma_{R}^{6}}\right) .
\end{aligned}
$$

Considering the mean, all variables are deterministic except $R_{\check{x}_{1} x_{2}}(0)$, and $E\left\{R_{\check{x}_{1} x_{2}}(0)-R_{\check{r} s}(0)\right\}=0$. Then it leads to

$$
E\left\{\frac{\partial^{2} \ln p}{\partial \varepsilon^{2}}\right\}=-\frac{\sigma_{R}\left(\partial^{2} \sigma_{R} / \partial \varepsilon^{2}\right)-\left(\partial \sigma_{R} / \partial \varepsilon\right)^{2}+\left(\partial R_{\check{r} s}(0) / \partial \varepsilon\right)^{2}}{\sigma_{R}^{2}}
$$

With the expression of $R_{\check{r} s}(0)$ in (13), and the definition of $\sigma_{R}$ in (26), the different derivatives are

$$
\begin{aligned}
\frac{\partial R_{\check{r} s}(0)}{\partial \varepsilon}= & -\frac{2 E_{s}}{\pi \varepsilon^{2} B T} \sin \left(\frac{\pi \varepsilon B T}{2}\right) \sin \left(\pi \varepsilon f_{0} T\right) \\
& +\frac{E_{s}}{\varepsilon} \cos \left(\frac{\pi \varepsilon B T}{2}\right) \sin \left(\pi \varepsilon f_{0} T\right) \\
& +\frac{2 E_{s} f_{0}}{\varepsilon B} \sin \left(\frac{\pi \varepsilon B T}{2}\right) \cos \left(\pi \varepsilon f_{0} T\right) \frac{\partial \sigma_{R}}{\partial \varepsilon} \\
= & \frac{E_{s} \sigma^{2}}{2 \sigma_{R}} \frac{\partial^{2} \sigma_{R}}{\partial \varepsilon^{2}}=-\frac{\left(E_{s} \sigma^{2}\right)^{2}}{4 \sigma_{R}^{3}} .
\end{aligned}
$$


Then (C.25) becomes

$$
\begin{aligned}
E\left\{\frac{\partial^{2} \ln p}{\partial \varepsilon^{2}}\right\}=- & -E_{s}^{2} \sigma^{4} / 2+\left(E_{s}^{2} \sigma_{R}^{2} / \varepsilon^{2}\right) \\
& \times\left((2 / \pi \varepsilon B T) \sin (\pi \varepsilon B T / 2) \sin \left(\pi \varepsilon f_{0} T\right)\right. \\
& -\cos (\pi \varepsilon B T / 2) \sin \left(\pi \varepsilon f_{0} T\right) \\
& \left.-\left(2 f_{0} / B\right) \sin (\pi \varepsilon B T / 2) \cos \left(\pi \varepsilon f_{0} T\right)\right)^{2} \\
/ & \left.\left((2+\varepsilon) E_{s} \sigma^{2}+T \sigma^{4}\right)^{2}\right) .
\end{aligned}
$$

The CR bound is finally written as a function of the SNR,

$$
\begin{aligned}
\mathrm{CR}= & {[(2+\varepsilon) \mathrm{SNR}+1]^{2} } \\
& /\left(T \mathrm { SNR } ^ { 2 } [ ( 2 + \varepsilon ) \mathrm { SNR } + 1 ] \left(\left(2 / \pi \varepsilon^{2} B T\right) \sin (\pi \varepsilon B T / 2)\right.\right. \\
& \left.\times \sin \left(\pi \varepsilon f_{0} T\right)-(1 / \varepsilon) \cos (\pi \varepsilon B T / 2)\right) \sin \left(\pi \varepsilon f_{0} T\right) \\
& \left.\left.-\left(2 f_{0} / B \varepsilon\right) \sin (\pi \varepsilon B T / 2) \cos \left(\pi \varepsilon f_{0} T\right)\right)^{2}-\mathrm{SNR}^{2} / 2\right) \\
= & (2+\varepsilon) \mathrm{SNR}+1]^{2} \\
& /\left(T \mathrm { SNR } { } ^ { 2 } [ ( 2 + \varepsilon ) \mathrm { SNR } + 1 ] \left(\left(2 / \pi \varepsilon^{2} B T\right) \sin (\pi \varepsilon B T / 2)\right.\right. \\
& \times \sin \left(\pi \varepsilon f_{0} T\right)-\left(\left(B+2 f_{0}\right) / 2 B \varepsilon\right) \sin \left(\pi \varepsilon\left(B / 2+f_{0}\right) T\right) \\
& \left.\left.+\left(\left(B-2 f_{0}\right) / 2 B \varepsilon\right) \sin \left(\pi \varepsilon\left(B / 2-f_{0}\right) T\right)\right)^{2}-\mathrm{SNR}^{2} / 2\right) .
\end{aligned}
$$

\section{REFERENCES}

[1] Y. Doisy, L. Deruaz, S. P. Beerens, and R. Been, "Target Doppler estimation using wideband frequency modulated signals," IEEE Transactions on Signal Processing, vol. 48, no. 5, pp. 1213-1224, 2000.

[2] F. S. Foster, P. N. Burns, D. H. Simpson, S. R. Wilson, D. A. Christopher, and D. E. Goertz, "Ultrasound for the visualization and quantification of tumor microcirculation," Cancer and Metastasis Reviews, vol. 19, no. 1-2, pp. 131-138, 2000.

[3] E. Brusseau, J. Fromageau, N. G. Rognin, P. Delachartre, and D. Vray, "Investigating elastic properties of soft biological tissues," IEEE Engineering in Medicine and Biology Magazine, vol. 21, no. 4, pp. 86-94, 2002.

[4] A. Pesavento, C. Perrey, M. Krueger, and H. Ermert, "A timeefficient and accurate strain estimation concept for ultrasonic elastography using iterative phase zero estimation," IEEE Transactions on Ultrasonics, Ferroelectrics, and Frequency Control, vol. 46, no. 5, pp. 1057-1067, 1999.

[5] Md. R. Sharif and S. S. Abeysekera, "Efficient wideband signal parameter estimation using combined narrowband and wideband ambiguity functions," in Proceedings of IEEE Pacific RIM Conference on Communications, Computers, and Signal Processing (PACRIM '03), vol. 1, pp. 426-429, Victoria, BC, Canada, August 2003.
[6] Q. Jin, K. M. Wong, and Z.-Q. Luo, "The estimation of time delay and Doppler stretch of wideband signals," IEEE Transactions on Signal Processing, vol. 43, no. 4, pp. 904-916, 1995.

[7] G. Giunta, "Fast estimators of time delay and doppler stretch based on discrete-time methods," IEEE Transactions on Signal Processing, vol. 46, no. 7, pp. 1785-1797, 1998.

[8] K. M. Wong, Z.-Q. Luo, and Q. Jin, "Design of optimum signals for the simultaneous estimation of time delay and Doppler shift," IEEE Transactions on Signal Processing, vol. 41, no. 6, pp. 2141-2154, 1993.

[9] S. R. Dooley and A. K. Nandi, "Adaptive time delay and Doppler shift estimation for narrowband signals," IEE Proceedings: Radar, Sonar and Navigation, vol. 146, no. 5, pp. 243250, 1999.

[10] R. N. Bracewell, The Fourier Transform and Its Application, McGraw-Hill, New York, NY, USA, 2nd edition, 1986.

[11] A. Grennberg and M. Sandell, "Estimation of subsample time delay differences in narrowband ultrasonic echoes using the Hilbert transform correlation," IEEE Transactions on Ultrasonics, Ferroelectrics, and Frequency Control, vol. 41, no. 5, pp. 588-595, 1994.

[12] G. Arfken, Mathematical Methods for Physicists, Academic Press, Orlando, Fla, USA, 3rd edition, 1985.

[13] A. J. Weiss and E. Weinstein, "Fundamental limitations in passive time delay estimation- part 1: narrow-band systems," IEEE Transactions on Acoustics, Speech, and Signal Processing, vol. 31, no. 2, pp. 472-486, 1983.

[14] B. Friedlander, "On the Cramer-Rao bound for time delay and Doppler estimation," IEEE Transactions on Information Theory, vol. 30, no. 3, pp. 575-580, 1984.

[15] A. Dogandžić and A. Nehorai, "Cramer-Rao bounds for estimating range, velocity, and direction with an active array," IEEE Transactions on Signal Processing, vol. 49, no. 6, pp. 1122$1137,2001$.

Jérémie Fromageau was born in Orléans, France, in 1975 . He received the M.S. degree in physical acoustics from the University Denis Diderot, Paris 7, in 1999, and the Ph.D. degree in medical image processing from INSA Lyon, in 2003, both in France. He is currently a Postdoctoral Fellow in the Laboratory of Biorheology and Medical Ultrasonics, University of Montreal Hospital. His research interests include signal and image processing applied to medical ultrasound imaging, elastography, and high-frequency imaging.

Hervé Liebgott was born in France in 1979. He received the M.S degree in 2002 in electrical engineering and in acoustics and a Ph.D. degree in 2005 in acoustics from the Institut National des Sciences Appliquées (INSA), Lyon, France. He is currently an Associate Professor at the Electrical Engineering and Industrial Informatics Department at the IUTB, Universite Lyon1 and a Researcher at the Center for Research and Applications in Image and Signal Processing (CREATIS). His research interests include signal and image processing applied to ultrasonic imaging and more particularly elastography.
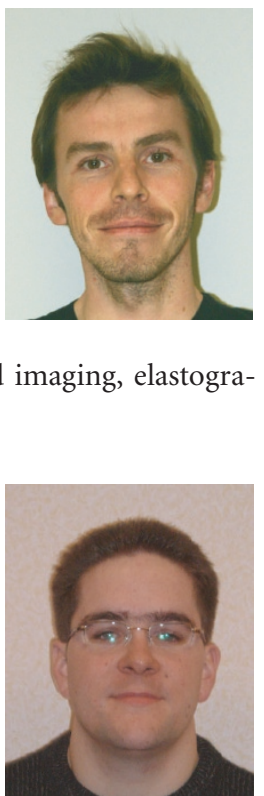
Elisabeth Brusseau is a Biomedical Engineer from the Technological University of Compiègne in France. She received an M.S. degree in biomedical engineering in 1997 in Compiègne and a Ph.D. degree in ultrasound imaging in the CREATIS laboratory in Lyon in 2000. In 2001, she completed a postdoctoral training period at the experimental echocardiography directed by A. F. W. van der Steen in Rotterdam, the Nether-

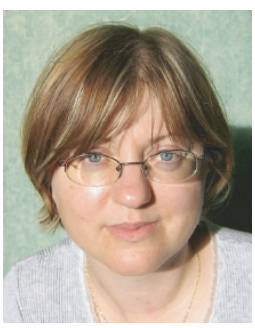
lands. She is now a CNRS Researcher at the CREATIS laboratory and her research focuses on elastography and ultrasound signal and image processing.

Didier Vray was born in Saint-Etienne, France, in 1959. He received his B.S.E. degree in electrical engineering from SaintEtienne University in 1981 and M.S. degree in applied computer sciences from the Institut National des Sciences Appliquées (INSA), Lyon, France, in 1984. He received the Ph.D. degree from INSA-Lyon, in 1989, for a work in acoustics and signal processing. He is currently a Professor of signal processing and computer sciences at INSA-Lyon. Since he joined the research laboratory CREATIS, his main research interests include ultrasound medical imaging, elastography, and highfrequency imaging.

Philippe Delachartre received in 1990 his M.S. degree and in 1994 a Ph.D. degree, both in signal and image processing in acoustics from the National Institute for Applied Sciences of Lyon (INSA-Lyon, France). Since 1995, he worked as an Associate Professor at the Electrical Engineering Department of the INSA-Lyon and Researcher at the Center for Research and Applications in Image and Signal Processing

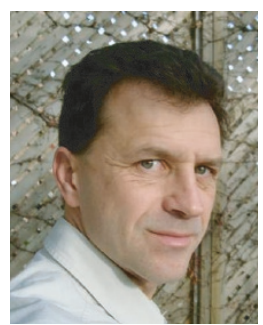
(CREATIS). His research interests include the image formation modeling and the parametric imaging applied to the field of medical ultrasound imaging. 